\title{
Comparison of the Lumbosacral Manipulation Method Versus Knee Exercises Alone in Patients with Patellofemoral Pain Syndrome
}

\author{
Sirous Azizi (iD ${ }^{1}$, Zahra Rezasoltani (iD) ${ }^{1}$, Elaheh Shirzadi (iD) ${ }^{1,}$, , Afsaneh Dadarkhah ${ }^{1}{ }^{1}$ and Morvarid \\ Elahi (iD ${ }^{2}$ \\ ${ }^{1}$ Aja University of Medical Sciences, Tehran, Iran \\ ${ }^{2}$ Tehran University of Medical Sciences, Tehran, Iran \\ "Corresponding author: Aja University of Medical Sciences, Tehran, Iran. Email: elaheh_shirzady@yahoo.com
}

Received 2020 October 02; Accepted 2020 October 26.

\begin{abstract}
Background: Patellofemoral Pain Syndrome (PFPS) is one of the most common diagnoses made for patients referred to physi$\mathrm{cal} /$ rehabilitation and orthopedic clinics. The most common symptom of PFPS is a diffuse pain in front of either one or both knees exacerbated by intense activity, kneeling, squatting, climbing, and weakness of quadriceps muscle. Lumbosacral manipulation and knee exercises are the most commonly used methods in physical medicine with no major side effects.

Objectives: The current study aimed to compare the efficacy of the lumbosacral manipulation technique with knee exercises versus sole knee exercises in patients with PFPS. Study Design was Randomized Controlled Clinical Trial.

Methods: In this randomized controlled clinical trial, 30 patients (18 females and 12 males) diagnosed with PFPS were divided into two groups of study and control, each with 15 subjects. Those in the intervention group received a single bilateral lumbosacral manipulation after ruling out any contraindication for lumbosacral manipulation by lumbosacral radiography. Quality of life, gait, quadriceps muscle strength, keen pain improvement, and range of motion were evaluated at the beginning and four weeks following the knee strengthening exercises for all participants.

Results: The mean age of participants was $34 \pm 5$ years. The difference between the groups concerning the swing phase of walking and quadriceps muscle strength was in both groups was investigated following providing interventions.

Conclusions: The manipulation technique had a greater effect on improving the function of patients with PFPS compared to therapeutic knee exercises.
\end{abstract}

Keywords: Manipulative Therapy, Therapeutic Exercises of the Knee, Patellofemoral Pain Syndrome, Lumbosacral Region

\section{Background}

Patellofemoral Pain Syndrome (PFPS), also known as runner's knee or jumper's knee, is one of the most common diagnoses made for patients referred to physical/rehabilitation and orthopedic clinics. Diffused pain in one or both of the knees is the most common symptom of PFPS. In most cases, the pain is exacerbated by intense activity, kneeling, squatting, climbing, and weakness of quadriceps muscle. However, the symptoms can be controlled by resting the knee as much as possible, decreasing activity level, and physical therapy (1-5).

While the exact cause of PFPS is unclear, it's believed to be caused by changes in the patellofemoral joint biomechanics due to over-use and damage to the joint such as internal rotation of the femur, genu varum, tibial torsion, subtalar joint pronation, muscle imbalance, previous knee trauma, declined muscle flexibility, and quadriceps mus- cle strength.

Patients suffering from PFPS may experience major physical disabilities, which significantly declines the quality of life (QoL) $(1,3,6)$. Therefore, determining the most plausible pain mechanism is of crucial importance to develop proper treatments with the highest efficacy and lowest possible complications. In this context, various methods have been used to determine the plausible mechanisms of knee pain, including clinical examination, medical imaging, as well as arthroscopy, each with their own specificity and sensitivity (7).

The lumbosacral manipulation and knee exercises are the most widely used treatment in physical medicine to treat PFPS that carry no major side effects (8-11). Manipulative therapy was first introduced in Europe back in the 4th century. Since the19th century, this technique has been rapidly expanding around the world, particularly in the 
fields of osteopathy and chiropractic $(12,13)$. The science of manipulation is based on the fact that even minor disorders of the central nervous system, particularly the peripheral nerves, can cause severe vague pain in lower extremities (14).

Although the main mechanisms of manipulation techniques are not clear yet, it's believed that they may lead to neuroplastic changes by the biomechanical recovery of the corresponding joint (13). Physicians readjust the vertebrae to the correct position by subtle movements, which dramatically reduces the pain from nerve impingement (15, 16). Lumbosacral manipulation, which has been approved by the US Food and Drug Administration, is reported to be beneficial for some patients with acute and chronic pain such as lumbar disc diseases $(15,17)$. Only a trained professional should provide Lumbosacral manipulation.

\section{Objectives}

Since there are controversies regarding the effectiveness of lumbosacral manipulation therapy (1, 18-21), the current study aimed to compare lumbosacral manipulation therapy with knee exercises versus knee exercises alone in patients with PFPS.

\section{Methods}

In this randomized controlled clinical trial, all patients diagnosed with PFPS admitted to the Emam Reza hospital in Tehran (Iran) between 2017 and 2019 were included. After receiving the study approval by the Institutional Review Board at the AJA University of Medical Sciences and obtaining written informed consent from all participants, eligible patients were divided into two groups of study or control. Data were collected using a checklist and questionnaire. The inclusion criterion was referring to the physical medicine and rehabilitation clinics of the Emam Reza Hospital with PFPS diagnosis. Exclusion criteria were being diagnosed with radiculopathy, tibiofemoral degenerative joint diseases (grade 2 and above based on the Kellgren-Lawrence radiographic grading), existing pes planus, and history of lumbar disc herniation, surgery, and knee trauma. The eligible patients were divided into two groups, each with 15 subjects. The study group received the lumbosacral manipulation with the knee exercises, and the control group received the knee exercises alone.

Radiographic examinations, including anteriorposterior, lateral, and patellar views of the knee, were performed for all participants at the beginning of the study. Anterior-posterior and lateral views of the lumbosacral were also obtained for all participants.
All individuals in the study group received a single bilateral lumbosacral manipulation after ruling out any contraindication for lumbosacral manipulation by lumbosacral radiography. The manual movements were performed by a trained professional at the level of L2 to S1 (the quadriceps and hamstrings muscles' neuronal levels). Then, both groups participated in the therapeutic strengthening knee exercise program (included the straight leg raise, quadriceps isometric, cuff, and hamstring stretching exercises) for 4 weeks (twice a day, every day of the week). It should be noted that individuals were not allowed to perform activities that could trigger knee pain during the program. All exercise techniques were based on Therapeutic Exercise, Foundations, and Techniques by Carolyn Kinser (22).

Gait analysis (including cadence, foot pressure, single support time, and double support time), measuring the range of motion of the knee, assessment of quadriceps muscle strength by superficial electromyography, Visual Analog Scale (VAS), and the Knee injury and Osteoarthritis Outcome Score (KOOS) questionnaires were used to collect information before providing the interventions, during the second session, and four weeks after knee strengthening exercises.

To assess improvement in pain severity, VAS was used in two measurement sessions (23). To assess the knee pain, symptoms, function in daily living, function in sport and recreation, and knee-related quality of life, the KOOS worldwide questionnaire was completed in two measurement sessions by all participants. The KOOS includes 42 patient-centered items on five patient-related subscales: Pain (9 items), symptoms (stiffness, etc.) related to the disease ( 7 items), daily activities (climbing the stairs, standing, etc.) (17 items), sports and recreational activities (jumping and running) (5 items), and knee-related QoL (4 items). Each item has five possible answers on a Likert scale. The score of each subscale ranges from zero to 4 . Therefore, the total score of KOOS ranges from zero ("No Problems") to 100 (Extreme Problems) (24).

The Kellgren-Lawrence radiographic grading system uses radiological evidence of osteophytes presence and reduction of articular space to categorize patients into five groups based on the severity of the pain, as follows: grade 0 (none); grade1(doubtful narrowing of the joint space with possible osteophyte formation); grade 2 (possible narrowing of the joint space with definite osteophyte formation); grade 3 (definite narrowing of joint space, moderate osteophyte formation, some sclerosis, and possible deformity of bony ends); grade 4 (osteophyte formation, severe narrowing of the joint space with marked sclerosis, and definite deformity of the bone end) (25). Most of the recent osteoarthritis-related studies have used this grading sys- 
tem to diagnose osteoarthritis or to determine the severity of joint involvement.

\subsection{Data Analysis}

Data analysis was performed using SPSS version 24 . Quantitative data are described using mean and standard deviation. While frequency was used to describe qualitative data. The Kolmogorov-Smirnov test was applied to test for a normal distribution. Quantitative variables were analyzed using t-test and ANOVA. Categorical variables were compared using the Chi-square test. Statistical significance was considered when P value $<0.05$.

\section{Results}

In total 37 patients enrolled to participate in the present study, that seven were excluded due to the insufficiency of data. The remaining 30 patients [ 18 females and 12 males] had a mean age of $34 \pm 5$ years. There was no significant difference concerning the variables of age, gender, height, weight, and duration of the disease in both groups. The demographic characteristics of the participants are described in Table 1.

\begin{tabular}{lccc}
\hline \multicolumn{3}{l}{ Table 1. Demographic Characteristics of Participants Separated by the Group } \\
\hline & Study Group & Control Group & P Value \\
\hline Age (years) & $35.0 \pm 4.2$ & $34.1 \pm 3.6$ & 0.52 \\
Gender (female) & $9(60 \%)$ & $9(60 \%)$ & 1.00 \\
Height $(\mathbf{c m})$ & $169 \pm 12$ & $170 \pm 9$ & 0.81 \\
Weight $(\mathbf{k g})$ & $70.4 \pm 15$ & $72.0 \pm 16$ & 0.78 \\
$\begin{array}{l}\text { Duration of disease } \\
\text { (months) }\end{array}$ & $6.0 \pm 4.1$ & $7.6 \pm 2.7$ & 0.20 \\
\hline
\end{tabular}

No significant difference was observed concerning the VAS and KOOS indexes of participants in both groups, before and 4 weeks after providing the intervention (Table 2).

Based on the results of the gait analysis, a significant difference was observed in the swing phase of walking between the two groups four weeks after providing the intervention. Also, an association was observed between foot pressure and the method of lumbosacral manipulation (Table 3).

No significant difference was found concerning the knee range of motions. However, quadriceps muscle strength was increased in the study group (Table 4).

\section{Discussion}

Various risk factors are suggested for PFPS, including gender, existing pes plan us, and extreme activities. Ado- lescents and young people are at greater risk of PFPS, however, the elderly also may experience PFPS. It's more common among females than males, probably due to weaker muscles and wider pelvic angle. Also, those with pes planus are more vulnerable to PFPS syndrome because of the additional pressure on their knees. Also, overuse of knees (e.g. running and jumping sports) put repetitive stress on the joints, which enhances the risk of developing PFPS following a knee injury $(26,27)$.

There was no difference between the participants of the present study concerning the demographic characteristics. Moreover, each group comprised of six males and nine females, as we expected. Nevertheless, there was no statistically significant gender difference between the two groups $(\mathrm{P}=1.00)$.

Findings about the efficacy of lumbosacral manipulation therapy are controversial (1, 18-21, 28-30). Miller et al., in a study on 18 PFPS patients, reported that Kinesio taping was more effective than lumbosacral manipulation (18). This dissimilarity can be attributed to the differences caused by the second method used in the treatment of patients. Grindstaff et al., in a study on 48 PFPS patients, showed that lumbosacral manipulation didn't have any immediate effect on quadriceps muscle strength (19). However, in the present study, a statistically significant difference was observed in quadriceps muscle strength based on the surface electromyography and swing phase of walking after 4 weeks in the study group compared to the control group. This finding is consistent with previous reports. Crowell et al. (1) and Iveronet al., reported that Lumbopelvic manipulation was effective in $57 \%$ and $45 \%$ of PFPS patients, respectively $(1,20)$. Although previous studies reported some complications and risks after providing Lumbopelvic manipulation, in the present study no complication was observed among participants $(11,31)$.

In conclusion, this study demonstrated that using manipulation caused significant improvements in PFPS patients compared to the sole therapeutic exercise. Accordingly, incorporating this method can improve the functions of PFPS patients.

\section{Footnotes}

Authors' Contribution: Study concept and design: Sirous Azizi, Acquisition of data: Elaheh Shirzadi, Analysis, and interpretation of data: Morvarid Elahi, Drafting of the manuscript: Afsaneh Ddarkhah, Critical revision of the manuscript for important intellectual content: Sirous Azizi, Statistical analysis: Morvarid Elahi, Administrative, technical, and material support: Sirous Azizi, Study supervision: Sirous Azizi. 
Azizi S et al.

\begin{tabular}{|c|c|c|c|c|c|}
\hline & \multicolumn{2}{|c|}{ Study Group } & \multicolumn{2}{|c|}{ Control Group } & \multirow[t]{2}{*}{ P Value } \\
\hline & Beginning of the Study & After 4 Weeks & Beginning of the Study & After 4 Weeks & \\
\hline VAS score index & $5 \pm 1.1$ & $3.6 \pm 1.2$ & $5 \pm 1.3$ & $3.7 \pm 1.2$ & 0.39 \\
\hline KOOS score index for the knee pain & $65 \pm 12$ & $76 \pm 13$ & $65 \pm 14$ & $73 \pm 10$ & 0.97 \\
\hline KOOS score index for symptoms & $79 \pm 16$ & $81 \pm 11$ & $77 \pm 13$ & $82 \pm 10$ & 0.68 \\
\hline KOOS score index for function in daily living & $68 \pm 11$ & $77 \pm 4$ & $72 \pm 12$ & $80 \pm 13$ & 0.42 \\
\hline KOOS score index for function in sport and recreation & $56 \pm 15$ & $67 \pm 13$ & $59 \pm 4$ & $68 \pm 2$ & 0.53 \\
\hline KOOS score index for knee-related quality of life & $59 \pm 14$ & $60 \pm 10$ & $60 \pm 13$ & $65 \pm 10$ & 0.51 \\
\hline
\end{tabular}

Abbreviations: VAS, Visual Analog Scale; KOOS, Knee injury and Osteoarthritis Outcome Score.

Table 3. Comparison of the Mean of Gait Analysis Separated by the Group

\begin{tabular}{|c|c|c|c|c|c|}
\hline & \multicolumn{2}{|l|}{ Study Group } & \multicolumn{2}{|c|}{ Control Group } & \multirow[t]{2}{*}{ PValue } \\
\hline & Beginning of the Study & After 4 Weeks & Beginning of the Study & After 4 Weeks & \\
\hline Swing phase of walking (\%) & $33.4 \pm 3$ & $33 \pm 2$ & $33.6 \pm 2$ & $31 \pm 3$ & 0.03 \\
\hline Stance phase of walking (\%) & $66 \pm 3$ & $66 \pm 2.5$ & $65.4 \pm 1.6$ & $68.1 \pm 2.8$ & 0.61 \\
\hline Index of cadence (step/min) & $52 \pm 4$ & $54 \pm 4$ & $50 \pm 4$ & $52 \pm 4$ & 0.45 \\
\hline Foot pressure $\left(\mathbf{n} / \mathrm{cm}^{2}\right)$ & $28 \pm 1.6$ & $27 \pm 1.1$ & $29.6 \pm 2.6$ & $29.6 \pm 2.5$ & 0.05 \\
\hline Single support time (second) & $0.33 \pm 0.03$ & $0.33 \pm 0.02$ & $0.32 \pm 0.02$ & $0.32 \pm 0.02$ & 0.23 \\
\hline \multirow[t]{3}{*}{ Double support time (second) } & $0.33 \pm 0.05$ & $0.32 \pm 0.03$ & $0.34 \pm 0.03$ & $0.33 \pm 0.05$ & 0.56 \\
\hline & \multicolumn{2}{|c|}{ Study group } & \multicolumn{2}{|c|}{ Control group } & P Value \\
\hline & Beginning of the Study & Week 4 & Beginning of the Study & Week 4 & \\
\hline Extension rang of motion (degree) & 0.0 & 0.0 & $0.6 \pm 2.5$ & $2.3 \pm 4.9$ & 0.32 \\
\hline Flexion range of motion (degree) & $108 \pm 5$ & $108 \pm 5$ & $112 \pm 6$ & $112 \pm 6$ & 0.11 \\
\hline Quadriceps muscle strength (mV) & $596 \pm 139$ & $873 \pm 119$ & $606 \pm 133$ & $720 \pm 138$ & 0.003 \\
\hline
\end{tabular}

\section{Clinical Trial Registration Code:}

IRCT20180416039324N1.

Conflict of Interests: The authors declare no conflict of interest.

Ethical Approval: IR.AJAUMS.REC.1397.006.

Funding/Support: AJA university of medical sciences.

\section{References}

1. Crowell MS, Wofford NH. Lumbopelvic manipulation in patients with patellofemoral pain syndrome.J Man Manip Ther. 2012;20(3):11320. doi: 10.1179/2042618612Y.0000000002. [PubMed: 23904749]. [PubMed Central: PMC3419567].

2. Lack S, Barton C, Vicenzino B, Morrissey D. Outcome predictors for conservative patellofemoral pain management: a systematic review and meta-analysis. Sports Med. 2014;44(12):1703-16. doi: 10.1007/s40279-014-0231-5. [PubMed: 25100644].

3. Motealleh A, Gheysari E, Shokri E, Sobhani S. The immediate effect of lumbopelvic manipulation on EMG of vasti and gluteus medius in athletes with patellofemoral pain syndrome: A randomized controlled trial. Man Ther. 2016;22:16-21. doi: 10.1016/j.math.2016.02.002. [PubMed: 26995778].

4. Matthews M, Rathleff MS, Claus A, McPoil T, Nee R, Crossley K, et al. Can we predict the outcome for people with patellofemoral pain? A systematic review on prognostic factors and treatment effect modifiers. BrJ Sports Med. 2017;51(23):1650-60. doi: 10.1136/bjsports-2016-096545. [PubMed: 27965435].

5. Esculier JF, Bouyer LJ, Dubois B, Fremont P, Moore L, McFadyen $\mathrm{B}$, et al. Is combining gait retraining or an exercise programme with education better than education alone in treating runners with patellofemoral pain?A randomised clinical trial. $\mathrm{Br} J$ Sports Med. 2018;52(10):659-66. doi: 10.1136/bjsports-2016-096988. [PubMed: 28476901].

6. Behrangrad S, Kamali F. Comparison of ischemic compression and lumbopelvic manipulation as trigger point therapy for patellofemoral pain syndrome in young adults: A double-blind randomized clinical trial. J Bodyw Mov Ther. 2017;21(3):554-64. doi: 10.1016/j.jbmt.2016.08.007. [PubMed: 28750964].

7. Solomon DH, Simel DL, Bates DW, Katz JN, Schaffer JL. The rational clinical examination. Does this patient have a torn meniscus or ligament of the knee? Value of the physical examination. 
JAMA. 2001;286(13):1610-20. doi: 10.1001/jama.286.13.1610. [PubMed: 11585485].

8. Stefanick GF. Low-tech rehabilitation of bilateral patellofemoral knee pain in a runner: a case study. The Journal of the Canadian Chiropractic Association. 2004;48(4):259.

9. Jayaseelan DJ, Courtney CA, Kecman M, Alcorn D. Lumbar manipulation and exercise in the management of anterior knee pain and diminished quadriceps activation following acl reconstruction: a case report. Int J Sports Phys Ther. 2014;9(7):991-1003. [PubMed: 25540713]. [PubMed Central: PMC4275202].

10. Cook C, Shah A, Pietrobon R. Lumbopelvic manipulation for treatment of patients with patellofemoral pain syndrome: development of a clinical predication rule. The Journal of orthopaedic and sports physical therapy. 2008;38(11):722.

11. Oliphant D. Safety of spinal manipulation in the treatment of lumbar disk herniations: a systematic review and risk assessment. J Manipulative Physiol Ther. 2004;27(3):197-210. doi: 10.1016/j.jmpt.2003.12.023. [PubMed: 15129202].

12. Pettman E. A history of manipulative therapy. J Man Manip Ther. 2007;15(3):165-74. doi: 10.1179/106698107790819873. [PubMed: 19066664]. [PubMed Central: PMC2565620].

13. Colloca CJ, Keller TS, Gunzburg R, Vandeputte K, Fuhr AW. Neurophysiologic response to intraoperative lumbosacral spinal manipulation. J Manipulative Physiol Ther. 2000;23(7):447-57. doi: 10.1067/mmt.2000.108822. [PubMed:11004648].

14. Haavik H, Murphy B. The role of spinal manipulation in addressing disordered sensorimotor integration and altered motor control. J Electromyogr Kinesiol. 2012;22(5):768-76. doi: 10.1016/j.jelekin.2012.02.012. [PubMed: 22483612].

15. Sutlive TG, Golden A, King K, Morris WB, Morrison JE, Moore JH, et al. Short-Term Effects of Trigger Point Dry Needling on Pain and Disability in Subjects with Patellofemoral Pain Syndrome. Int J Sports Phys Ther. 2018;13(3):462-73. [PubMed: 30038832]. [PubMed Central: PMC6044598].

16. Gorrell LM, Brown B, Lystad RP, Engel RM. Predictive factors for reporting adverse events following spinal manipulation in randomized clinical trials - secondary analysis of a systematic review. Musculoskelet Sci Pract. 2017;30:34-41. doi: 10.1016/j.msksp.2017.05.002. [PubMed: 28521180].

17. Farazdaghi MR, Motealleh A, Abtahi F, Panjan A, Sarabon N, Ghaffarinejad F. Effect of sacroiliac manipulation on postural sway in quiet standing: a randomized controlled trial. Braz J Phys Ther. 2018;22(2):120-6. doi: 10.1016/j.bjpt.2017.09.002. [PubMed: 28993042]. [PubMed Central: PMC5883953].

18. Miller J, Westrick R, Diebal A, Marks C, Gerber JP. Immediate effects of lumbopelvic manipulation and lateral gluteal kinesio taping on unilateral patellofemoral pain syndrome: a pilot study. Sports Health. 2013;5(3):214-9. doi: 10.1177/1941738112473561. [PubMed: 24427391]. [PubMed Central: PMC3658409].

19. Grindstaff TL, Hertel J, Beazell JR, Magrum EM, Kerrigan DC, Fan X, et al. Lumbopelvic joint manipulation and quadriceps activation of people with patellofemoral pain syndrome. J Athl Train. 2012;47(1):2431. doi: 10.4085/1062-6050-47.1.24. [PubMed: 22488227]. [PubMed Central: PMC3418111].

20. Iverson CA, Sutlive TG, Crowell MS, Morrell RL, Perkins MW, Garber
MB, et al. Lumbopelvic manipulation for the treatment of patients with patellofemoral pain syndrome: development of a clinical prediction rule. J Orthop Sports Phys Ther. 2008;38(6):297-309. discussion 309-12. doi: 10.2519/jospt.2008.2669. [PubMed: 18515959].

21. Sanders GD, Nitz AJ, Abel MG, Symons TB, Shapiro R, Black WS, et al. Effects of Lumbosacral Manipulation on Isokinetic Strength of the Knee Extensors and Flexors in Healthy Subjects: A Randomized, Controlled, Single-Blind Crossover Trial. J Chiropr Med. 2015;14(4):240-8. doi: 10.1016/j.jcm.2015.08.002. [PubMed: 26793035]. [PubMed Central: PMC4688558].

22. Kisner C, Colby LA. Therapeutic exercise: foundations and techniques. FA Davis company; 1994.

23. Hawker GA, Mian S, Kendzerska T, French M. Measures of adult pain: Visual Analog Scale for Pain (VAS Pain), Numeric Rating Scale for Pain (NRS Pain), McGill Pain Questionnaire (MPQ), Short-Form McGill Pain Questionnaire (SF-MPQ), Chronic Pain Grade Scale (CPGS), Short Form-36 Bodily Pain Scale (SF-36 BPS), and Measure of Intermittent and Constant Osteoarthritis Pain (ICOAP). Arthritis Care Res (Hoboken). 2011;63 Suppl 11:S240-52. doi: 10.1002/acr.20543. [PubMed: 22588748].

24. Roos EM, Lohmander LS. The Knee injury and Osteoarthritis Outcome Score (KOOS): from joint injury to osteoarthritis. Health Qual Life Outcomes. 2003;1:64. doi: 10.1186/1477-7525-1-64. [PubMed: 14613558]. [PubMed Central: PMC280702].

25. Kohn MD, Sassoon AA, Fernando ND. Classifications in Brief: Kellgren-Lawrence Classification of Osteoarthritis. Clin Orthop Relat Res. 2016;474(8):1886-93. doi: 10.1007/s11999-016-4732-4. [PubMed: 26872913]. [PubMed Central: PMC4925407].

26. Espi-Lopez GV, Arnal-Gomez A, Balasch-Bernat M, Ingles M. Effectiveness of Manual Therapy Combined With Physical Therapy in Treatment of Patellofemoral Pain Syndrome: Systematic Review. J Chiropr Med. 2017;16(2):139-46. doi: 10.1016/j.jcm.2016.10.003. [PubMed: 28559754]. [PubMed Central: PMC5440631].

27. Espi-Lopez GV, Serra-Ano P, Vicent-Ferrando J, Sanchez-Moreno-Giner $\mathrm{M}$, Arias-Buria JL, Cleland J, et al. Effectiveness of Inclusion of Dry Needling in a Multimodal Therapy Program for Patellofemoral Pain: A Randomized Parallel-Group Trial. J Orthop Sports Phys Ther. 2017;47(6):392-401. doi: 10.2519/jospt.2017.7389. [PubMed: 28504067].

28. Han L, Zhao P, Guo W, Wei J, Wang F, Fan Y, et al. Short-term study on risk-benefit outcomes of two spinal manipulative therapies in the treatment of acute radiculopathy caused by lumbar disc herniation: study protocol for a randomized controlled trial. Trials. 2015;16:122. doi: 10.1186/s13063-015-0634-0. [PubMed: 25872929]. [PubMed Central: PMC4380109].

29. Santilli V, Beghi E, Finucci S. Chiropractic manipulation in the treatment of acute back pain and sciatica with disc protrusion: a randomized double-blind clinical trial of active and simulated spinal manipulations. Spine J. 2006;6(2):131-7. doi: 10.1016/j.spinee.2005.08.001. [PubMed: 16517383].

30. Leininger B, Bronfort G, Evans R, Reiter T. Spinal manipulation or mobilization for radiculopathy: a systematic review. Phys Med Rehabil Clin N Am. 2011;22(1):105-25. doi: 10.1016/j.pmr.2010.11.002. [PubMed: 21292148].

31. Ernst E. Adverse effects of spinal manipulation: a systematic review. J R Soc Med. 2007;100(7):330-8. doi: 10.1177/014107680710000716. [PubMed: 17606755]. [PubMed Central: PMC1905885]. 